\title{
CIII. On the orbits in the field of a doublet
}

\section{Dorothy Wrinch}

To cite this article: Dorothy Wrinch (1922) CIII. On the orbits in the field of a doublet, Philosophical Magazine Series 6, 43:257, 993-1014, DOI: 10.1080/14786442208633951

To link to this article: http://dx.doi.org/10.1080/14786442208633951

$$
\text { 册 Published online: } 08 \text { Apr } 2009 .
$$

Submit your article to this journal

Џ Article views: 2

Q View related articles $₫$ 
CIII. On the Orbits in the Field of a Doublet. By Dorothr Wrinch, Fellow of Girton College, Cambridge, and Member of the Research Staff, University College, London*.

\section{Introductory.}

$7 \mathrm{YHE}$ present paper investigates in a somewhat systematic way the two-dimensional motion of a particle in the field due to a doublet. Several of the results are of course known already as isolated theorems, but the subject does not appear to have been treated by any writer in a comprehensive manner, and it is very difficult to obtain a general view of the motion of a particle around a doublet even in two dimensions by putting together the specific solutions found in dy namical treatises or memoirs. Some new results which may be of importance are arrived at in the present paper, for the doublet may be of many types : the analysis is equally appropriate to a magnetic pole moving under the influence of an elementary magnet or to an electric charge in the presence of an electric doub et. Physicists are now generally convinced that the structure of a neutral atom of an element follows the lines generally associated with the names of Rutherford and Bohr. Such an atom behaves towards an external electron sufficiently far away effectively like a doublet, and we have little knowledge of its capacity to attract and retain a stray electron and so form a negatively charged atom. In a vacuum tabe, where the atoms are continnally bombarded by electrons, we know that negatively charged atoms frequently occur; but the conditions conducive to the presence of a large number in cases in which their existence is chemically possible appear to be quite unknown.

The success of the quantum theory has generally established the fact that we must not expect to be able, by the use of classical dynamice, to determine the internal motions of the electrons actually constituting the atom; but if we recall the facts that

(a) the inverse square law of attraction between electric charges appears to remain valid at distances comparable with atomic dimensions,

(b) the essential need for the quantum type of dynamical specification only arises when an electron is "bound" or is already a constituent part of the atom,

we see that the motion of an external charge must, even * Conmunicated by the Author.

Phil. Mag. S. 6. Vol. 43. No. 257. May 1922. 
on this view, be governed by the formulæ of classical dynamics.

For these reasons the results of this paper should have some bearing on the conditions under which an electron originally moving in any way in the neighbourhood of an atom may be captured and relained. But in default of a more precise development of atomic theory, we have set out the results as solutions of a formal general dynamical problem in the theory of orbits, for the need for such a scheme of solution is recognized here as well as in electrical theory.

A doublet consisting of two equal and opposite chargesof electrical or other type-subject to the inverse square law of attraction admits a potential

$$
\mathrm{V}=-\mu \cos \theta / r^{2}
$$

at all external points. This involves both a radial force $R$ and a transverse force $\mathrm{T}$ on any unit particle in the tield of the doublet. $R$ is measured along $r$ increasing, and $T$ in the direction $\theta$ increasing. The values are given by

$$
\begin{aligned}
& \mathrm{R}=-\frac{\partial \mathrm{V}}{\partial r}=-2 \mu \cos \theta / r^{3}, \\
& \mathrm{~T}=-\partial \mathrm{V} / r \partial \theta=-\mu \sin \theta / r^{3} .
\end{aligned}
$$

The equations of the orbit of the particle are of the wellknown form

$$
\begin{array}{ll}
\ddot{r}-r \dot{\theta}^{2} & =\mathrm{R}, \\
1 / r d / d t\left(r^{2} \dot{\theta}\right) & =\mathrm{T} .
\end{array}
$$

Denoting $r^{2} \dot{\theta}$ by $h$-so that $m h$ is the angular momentum when the particle has mass $m,-$ we can show that

and also

$$
\begin{aligned}
h^{2} & =(-\mathrm{R}-\mathrm{T} d u / u d \theta) / h^{2}\left(u+d^{2} u / d \theta^{2}\right) \\
& =\mu(\sin \theta d u / d \theta+2 u \cos \theta) /\left(u+d^{2} u / d \theta^{2}\right) ; .
\end{aligned}
$$

These are obtained by the usual procedure of eliminating the time. The field of the donblet is symmetrical about the axis $\theta=0$, and we shall find it convenient to consider only those motions which begin on the side of the plane defined by

$$
\pi \geq \theta \geq 0
$$

for it is clear that other possible motions will merely be reflexions of these in the axis; their separate discussion is therefore unnecessary. 
The equation may be integrated in the form

$$
h^{2}=2 \mu\left(\cos \theta-\cos \theta_{1}\right)+h_{1}^{2},
$$

where $h$ takes the value $h_{1}$ when $\theta$ takes some specific value $\theta_{1}$. Now motion can only take place when $h$ is real, so that $h^{2}$ must not have negative values. It is therefore evident that no motion can occur when

$$
2 \mu \cos \theta_{1}-h_{1}^{2}>2 \mu,
$$

and in the critical case,

$$
2 \mu \cos \theta_{1}-h_{1}^{2}=2 \mu,
$$

the motion is restricted to the line $\theta=0$ and is given by

$$
\ddot{r}=-2 \mu / r^{3} \text {. }
$$

Motion along the axis of the Doublet.

We may now proceed to discuss these motions along the axis in some detail.

The integrated form of the equation (1) is

$$
\dot{r}^{2}=\mathrm{O}+2 \mu / \mathrm{r}^{2} \text {. }
$$

If the velocity $U$ at the point of projection $r=r_{1}$ is $+U_{1}$, then

$$
\frac{d r}{d t}=\mathrm{U}=+\sqrt{\mathrm{U}_{1}^{2}+2 \mu / r^{2}-2 \mu / r_{1}^{2}} .
$$

If $\mathrm{U}_{1}^{2}>2 \mu / r_{1}^{2}$, the particle describes the line $\theta=0$ in the directicn $r$ increasing, with a steadily decreasing velocity. Its velocity at $r=\infty$ is $\sqrt{\mathrm{U}_{1}{ }^{2}-2 \mu / r_{1}{ }^{2}}$. Further, integrating the equation again, the time is given as a function of $r$ in the lorm

$$
t-t_{1}=\left(\sqrt{\left(\mathrm{U}_{1}^{2}-2 \mu / r_{1}^{2}\right) r^{2}+2 \mu}-\sqrt{2 \mu}\right) /\left(\mathrm{U}_{1}{ }^{2}-2 \mu / r_{1}^{2}\right),
$$

and the time to infinity is infinite. Thus a particle with these conditions of projection will not leave the system in a finite time.

Tha case $\mathrm{U}_{1}^{2}=2 \mu / \mathrm{r}_{1}^{2}$ gives a similar result. The particle describes the line $\theta=0$ in the direction $r$ increasing with a steadily decreasing velocity, arriving there with zero velocity. The time is given by

$$
t-t_{1}=\left(r^{2}-r_{1}^{2}\right) / 2 \sqrt{2 \mu} ;
$$

so that, as before, an infinite time is required for the description of the line $\theta=0$. 
Now it may easily be seen that $+\sqrt{\frac{2 \mu}{r_{1}{ }^{2}}}$ is the smallest velocity of projection which causes a particle to recede from the doublet and ultimately to leave the system. When

$$
\mathrm{U}_{1}<\sqrt{ } 2 \mu / \boldsymbol{r}_{1}^{2} \text {, }
$$

the particle returns to the doublet. For since

$$
\dot{r}=\mathrm{U}=\sqrt{ }\left(\mathrm{U}_{1}{ }^{2}+2 \mu / r^{2}-2 \mu / r_{1}{ }^{2}\right), \ldots .
$$

$\mathrm{U}$ will vanish at $r=r_{2}$, where

$$
1 / r_{1}^{2}-1 / r_{2}^{2}=\mathrm{U}_{1}^{2} / 2 \mu
$$

the particle then reverses its direction and approaches the doublet. Integrating equation (4), we have

$$
t-t_{1}=r_{2}\left(\sqrt{{r_{2}{ }^{2}}^{2}-r_{1}^{2}}-\sqrt{r_{2}^{2}-r^{2}}\right) / \sqrt{2 \mu} \text {; }
$$

so that the time from $r=r_{1}$, at which $t=t_{1}$, to $r=r_{2}$, at which $t=t_{2}$, is

$$
r_{2} \sqrt{r_{2}^{2}-r_{1}^{2}} / \sqrt{2 \mu}
$$

the particle reverses its direction, and its velocity is now given by

$$
\dot{r}=-\sqrt{ }\left(2 \mu / r^{2}-2 \mu / r_{2}^{2}\right),
$$

and the time therefore by

$$
t-t_{2}=r_{2} \sqrt{r_{2}^{2}-r^{2}} / \sqrt{2 \mu} .
$$

The time to the origin is consequently $r_{2}^{2} / \sqrt{2 \mu}$. The particle therefore proceeds in the direction $r$ increasing for a

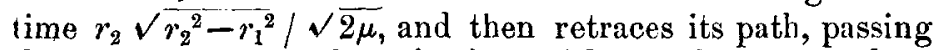
through its point of projection with a velocity equal to that with which it was projected and opposite in sign, arriving at the centre of force after a time

$$
\left(r_{2}^{2}+r_{2} \sqrt{r_{2}^{2}-r_{1}^{2}}\right) / \sqrt{2 \mu}
$$

with infinite velocity.

Considering next the cases when the particle is projected towards the origin from $r=r_{1}$ with velocity $\mathrm{U}_{1}$, we obtain the equation

$$
\dot{r}=-\sqrt{\mathrm{U}_{1}^{2}+2 \mu / r^{2}-2 \mu / r_{1}^{2}},
$$

and whatever the magnitude of $U_{1}$ the particle goes to the origin with steadily increasing velocity and arrives there with an infinite velocity. The times can be obtained 
by integrating the equation in the form

$$
\begin{aligned}
t-t_{1} & =\frac{r_{1}}{\mathrm{U}_{1}^{2} r_{1}^{2}-2 \mu}\left[\mathrm{U}_{1} r_{1}^{2}-\sqrt{\left(\mathrm{U}_{1}^{2} r_{1}^{2}-2 \mu\right) r^{2}+2 \mu r_{1}^{2}}\right] \\
& =\frac{r_{1}^{2}}{\mathrm{U}_{1} r_{1}^{2}+\sqrt{2 \mu r_{1}^{2}+\left(\mathrm{U}_{1}^{2} r_{1}^{2}-2 \mu\right) r^{2}}}
\end{aligned}
$$

thus the time to the origin is

$$
r_{1} /\left(\mathrm{U}_{1}+\sqrt{ } 2 \mu / r_{1}\right)
$$

giving $r_{1} / 2 \mathrm{U}_{1}$ in the case when $\mathrm{U}_{1}^{2}=2 \mu / r_{1}^{2}$. The particie can consequently be sent to the origin in any time however small if $U_{1}$ is sufficiently large, and attain an infinite velocity in the time; it is further evident that it can approach the doublet with an infinite velocity even if it is projected with zero velocity or a velocity away from the origin, however small its original distance from the origin, provided only that it is not projected away from the doublet at the point $r_{1}$ with as great a velocity as $\sqrt{2 \mu /{r_{1}^{2}}^{2}}$

As regards motion on the line $\theta=\pi$, the equation is

giving

$$
\ddot{r}=2 \mu / r^{3} \text {, }
$$

Thus

$$
\dot{r}=C-2 \mu / r^{2} \text {. }
$$

$$
\dot{r}=+\sqrt{\mathrm{U}_{1}^{2}-2 \mu / r^{2}+2 \mu / r_{1}^{2}}
$$

if $\mathrm{U}_{1}$ is the velocity away from the origin at $r=r_{1}$. Thus $\dot{r}$ increases as $r$ increases and has the value

$$
\sqrt{\mathrm{U}_{1}{ }^{2}+2 \mu / r_{1}}
$$

at infinity. The time equation can be obtained as before, and it is found that the time of transit of the line is infinite. If, however, the particle is projected towards the origin

$$
\dot{r}=-\sqrt{\mathrm{U}_{1}{ }^{2}-2 \mu / r^{2}+2 \mu / r_{1}^{2}}
$$

and vanishes at $r=r_{2}$ given by

the time to $r_{2}$ is

$$
\mathrm{U}_{1}{ }^{2}-2 \mu / r_{2}{ }^{2}+2 \mu / r_{1}^{2}=0 ;
$$

$$
r_{2} \sqrt{r_{1}^{2}-r_{2}^{2}} / \sqrt{2 \mu}
$$

and the particle recedes from the origin and disappears from the system, though only after an infinite time. Thus no conditions of projection in which $\mathrm{U}_{1}$ is finite keep the particle from steadily increasing its distance from the 
doublet. If it is projected towards the doublet, it goes to the point $r=r_{2}$ and then recedes to infinity; otherwise it moves away from it at once.

We have therefore two different types of motion, which are typical of the asymmetry of the field of force of the doublet. If the particle is in the line $\theta=\pi$, so long as the angular velocity is zero, no conditions of projection can prevent the particle from receding from the doublet with increasing speed, either immediately or after an interval

$$
2 r_{2} \sqrt{r_{1}^{2}-r_{2}^{2}} / \sqrt{2 \mu} \text {. }
$$

If, however, the particle is in the line $\theta=0$, so long as the angular velocity is zero, the particle does not leare the system unless projection is away from the origin and the velocity attains or exceeds the critical value $\sqrt{2 \mu} / r_{1}$. If the velocity of projection away from the origin is less than $\sqrt{2 \mu} / r_{1}$-and this includes the case of zero velocity or of projection towards the centre-the particle approaches the centre after an interval

$$
2 r_{2} \sqrt{r_{2}^{2}-r_{1}^{2}} / \sqrt{2 \mu}
$$

or directly, according as $\mathrm{U}_{1}>0$ or $\mathrm{U}_{1} \leq 0, r_{2}$ being given by

$$
1 / r_{2}^{2}=1 / r_{1}^{2}-U_{1}^{2} / 2 \mu
$$

and the particle arrives at the centre in a finite time with infinite velocity.

These conclusions lead to curious effects of electrical doublets in the presence of stray electrons, which have apparently not been completely realized. It is remarkable that these stray electrons, when placed on one half of the axis of the doublet, should arrive from any distance, however large or small, and bombard the doublet with very great momentum ; and that, on the other hand, they must leave the system altogether if they are on the other side of the axis. It follows that a necessary consequence of the field of force created by a doublet is a continuous bombardment on one side by any stray electrons, and a steady ejection of electrons from the system on the other side.

\section{General Motions.}

Thus far, we have considered only the case when particles are projected from some point on the axes of symmetry of the doublet, with no angular velocity. The angular 
momentum is given, in general, by the equation

$$
h^{2}=h_{1}^{2}+2 \mu\left(\cos \theta-\cos \theta_{1}\right) ; . . . .
$$

so that if $\mathrm{V}$ be the transverse velocity at any point, and in particular $V_{1}$ the transverse velocity at $\left(r_{1}, \theta_{1}\right)$,

$$
r^{2} V^{2}=r_{1}^{2} V_{1}^{2}+2 \mu\left(\cos \theta-\cos \theta_{1}\right) \text {. }
$$

Since $h^{2}$ cannot be negative, motion can only take place in the part of the plane in which

$$
\cos \theta \geq \cos \theta_{1}-h_{1}^{2} / 2 \mu \text {. }
$$

It is now evident that there is an important division of the possible orbits in the field of the doublet. For if the initial conditions are such that

$$
h_{1}{ }^{2}>2 \mu\left(1+\cos \theta_{1}\right),
$$

the condition that $h^{2}$ the square of the angular momentum is not negative is satisfied at all points of the plane, and the motion is therefore not restricted to any region of the plaue, If, however,

$$
-1 \leq \cos \theta_{1}-h_{1}^{2} / 2 \mu=m \leq 1,
$$

the expression for $h^{2}$ is positive or zero only when $\cos \theta-m$ is positive or zero ; and this plainly limits the motion to the sector of the plane (including the line $\theta=0$ ) bounded by the radius vectors $\theta=\alpha$ and $\theta=-\alpha$, where $m=\cos \alpha$. 'T'hus in this case, if $\cos \theta_{1}-h_{1}^{2} / 2 \mu$ lies between zero and unity, the motion is restricted to the sector $\theta= \pm \alpha$, and $\alpha$ is not greater than $\pi / 2$. If $h_{1}^{2} / 2 \mu-\cos \theta_{1}$ lies between zero and unity, the motion is restricted to a sector $\theta= \pm \alpha$, and in this case $a$ is not less than $\pi / 2$.

The initial transverse velocity of projection therefore determines the orbits to this extent. If

$$
r_{1}^{2} \nabla_{1}^{2} / 2 \mu>1+\cos \theta_{1}
$$

motion is possible all over the plane; if, however,

and

$$
r_{1}^{2} V_{1}^{2} / 2 \mu=\cos \theta_{1}-m
$$

$$
-1 \leq m=\cos \alpha \leq 1 \text {, }
$$

the motion is restricted to a sector $\theta= \pm \alpha$. Plainly, the larger $r_{1}^{2} V_{1}^{2}$ is, the larger is the sector; in the limiting case when $r_{1}^{2} V_{1}^{2} / 2 \mu=1+\cos \theta_{1}$, the sector is the whole plane.

This discussion exhausts all possible cases, since plainly we cannot have

$$
r_{1}^{2} V_{1}^{2} / 2 \mu<\cos \theta-1 \text {. }
$$


Characteristics of the Radial Motion : Reluction to the Case of Motion on the Axis of the Doublet.

Consider next the $(r, t)$ equation relating the radius vector to the time. We have

$$
\ddot{r}-r \dot{\theta^{2}}=\ddot{r}-h^{2} / r^{3}=-2 \mu \cos \theta / r^{3} .
$$

Consequently,

where

$$
\ddot{r}=-2 \mu m / r^{3},
$$

$$
m=\cos \theta_{1}-h_{1}^{2} / 2 \mu
$$

as before. Now, we have already discussed the equations

$$
\ddot{r}=-2 \mu / r^{3}, \quad \ddot{r}=+2 \mu / r^{3},
$$

which give the motion on the lines $\theta=0$ and $\theta=\pi$ respectively in the case when the particle has no angular momentum. The results in two cases can be roadily modified so as to cover the general case of motion-the first equation for the case when $m$ is positive, and the second for the case when $m$ is negative-by merely writing $2 \mu \mathrm{m}$ for $2 \mu$. In fact, so far as the behaviour of $r$ is concerned, the characteristics of orbits in "hich

$$
0 \geq r_{1}^{2} V_{1}^{2} / 2 \mu-\cos \theta_{1} \geq-1
$$

are similar to those on the line $\theta=0$; and the charucteristics of orbits in which

$$
r_{1}^{2} V_{1}^{2} / 2 \mu-\cos \theta_{1}>0
$$

are similar to those on the line $\theta=\pi$.

Thus, in the first set of cases, no conditions of projection with respect to the radial velocity can prevent the particle, either immediately or after a finite interval, receding from the origin with an ever-increasing velocity. In the second set of cases, it is only when the radial velocity is sufficiently big, viz.

$$
\mathrm{U}_{1} \geq+\sqrt{2 \mu m / r_{1}^{2}}
$$

that the particle leaves the system. In all other cases the particle arrives at the doublet in a finite time with an infinite velocity.

The difference in the characteristics of these two classes of orbits is of course not unexpected. For when $|\theta|>\pi / 2$ the radial force is repulsive, and for $|\theta|<\pi / 2$ attractive, On $|\theta|=\pi / 2$, it is zero. Consequently, if the particle has sufficient angular momentum to ass the line $|\theta|=\pi / 2$, and not sufficient to regain the sector $|\theta| \leq \pi / 2$ after one or more revolutions, nothing can prevent it from receding from 
the doublet, however great its initial radial velocity towards the doublet may be. If, however, the angular momentum is not great enough to enable the particle to get beyond the lines $|\theta|=\pi / 2$, or is great enough to enable it to regain the sector $|\theta| \leq \pi / 2$, it is only in the case of a sufficiently large radial velocity away from the origin that the particle can be prevented from approaching the centre (possibly alter an excursion away from it finite in time and extent) and bombarding the doublet with an infinite momentum after a finite time.

\section{Characteristics of the Orbits which lie in a Sector of the Plane.}

We may now discuss the variation in $\mathrm{V}$, the transverse velocity.

Suppose, first, that we limit ourselves to the cases when

$$
0<\cos \theta_{1}-r_{1}^{2} V_{1}^{2} / 2 \mu=\cos \alpha=m \leq 1,
$$

in which the motion is restricted between the lines $\theta= \pm \alpha$ and $\alpha \leq \pi / 2$.

$\mathrm{V}$ is always zero at all points of these bounding rays. Hence, except when $U$ the radial velocity also happens to vanish at a point on the bounding rays, at such a point the velocity of the particle is entirely along the radius vector. Consequently at such a point the orbit touches the bounding rays, except in the special case when the radial velocity is also momentarily evanescent.

Motion therefore takes place in general along a curve of wave form touching alternately the bounding rays $\theta= \pm \alpha$. If the particle is projected at $r_{1} \theta_{1}$ with velocities $\mathrm{U}=\mathrm{U}_{1} \geq 0$, $\mathrm{V}=\mathrm{V}_{1}$, the particle initially recedes from the doublet, touching the bounding rays alternately. If

$$
U_{1} \geq V\left(2 \mu \cos \alpha / r_{1}^{2}\right)
$$

it gets perpetually further from the origin. If, however,

$$
\mathrm{V}_{1}<\sqrt{ }\left(2 \mu \cos \alpha / r_{1}^{2}\right)
$$

the particle recedes from the origin along a curve of wave form, touching the rays alternately until it reaches a point $r_{2}$ given by

$$
1 / r_{1}^{2}-1 / r_{2}^{2}=\mathrm{U}_{1}^{2} / 2 \mu \cos \alpha .
$$

At this point its velocity along the radius vector is zero, and it therefore touches the circle $r=r_{2}$. Subsequently 
it approaches the doublet, converging on it along a wavelike path. In the case of an orbit of this type, the particle approaches the doublet with infinite velocity.

The equations of the orbits in these cases can be obtained either by integrating the original equation (1), which with the help of equation (2) may be written

$$
2(\cos \theta-\cos \alpha) d^{2} u / d \theta^{2}-\sin \theta d u / d \theta-2 u \cos \alpha=0
$$

or

$$
\sqrt{ }(\cos \theta-\cos \alpha) \frac{d}{d \theta}\left(\sqrt{ }(\cos \theta-\cos \alpha) \frac{d u}{d \theta}\right)-u \cos \alpha=0,
$$

or we may obtain them by using the fact that $\mathrm{U} / \mathrm{V}=d r / r d \theta$. Proceeding from this, we obtain

$$
-d u / u d \theta=d r / r d \theta=\dot{r} / r \dot{\theta}= \pm \frac{\sqrt{ }\left(u^{2}-u_{1}^{2}+\mathrm{U}_{1}^{2} / 2 \mu \cos \alpha\right)}{u \sqrt{ }(\cos \theta / \cos \alpha-1)}
$$

Hence

$$
\frac{d u}{\sqrt{\mathrm{U}_{1}^{2}+2 \mu u_{1}^{2}-2 \mu u_{1}^{2}} \cos \alpha}= \pm \frac{d \theta}{\sqrt{2 \mu \sqrt{\cos \theta-\cos \alpha}}} .
$$

Now if the orbit is being described initially with $r$ and $\theta$ increasing, $U / V>0$. This case needs the lower sign in the above equation.

Put

$$
\sin \theta / 2=\sin \alpha / 2 \operatorname{sn}(\psi, \sin \alpha / 2),
$$

where sn is the Jacobian elliptic function with modulus $\sin \alpha / 2$, and we obtain

$$
-\frac{d \theta}{\sqrt{ } 2 \mu \sqrt{\cos \theta-\cos \alpha}}=-\frac{\sqrt{ } 2 d \psi}{\sqrt{2 \mu}} .
$$

Further, putting

we have

$$
u=\sqrt{\mathrm{U}_{1}^{2} / 2 \mu \cos \alpha-u_{1}^{2}} \sinh \xi,
$$

$$
\frac{d u}{\sqrt{\mathrm{U}_{1}^{2}+2 \mu u^{2}-2 \mu u_{1}^{2}} \cos \alpha}=\frac{d \xi}{\sqrt{2 \mu \cos \alpha}} \text {. }
$$

Hence

$$
\frac{\xi-\xi_{0}}{\sqrt{\cos \alpha}}=-\sqrt{2} \psi
$$

where $\xi_{0}$ is some constant; and we obtain the equation of the orbit in terms of a parameter $\xi$ and constants $\alpha, \xi_{0}$ in the form

$$
\begin{aligned}
\sin \theta / 2 & =\sin (\alpha / 2) \operatorname{sn}\left[\left(\xi_{0}-\xi\right) / \sqrt{2 \cos \alpha}, \sin \alpha / 2\right], \\
u & =\sqrt{ }\left(\mathrm{U}_{1}^{2} / 2 \mu \cos \alpha-u_{1}^{2}\right) \sinh \xi
\end{aligned}
$$


or writing $\xi_{0}-\xi=\chi$,

$$
\begin{aligned}
\sin \theta / 2 & =\sin (\alpha / 2) \operatorname{sn}(\chi / \sqrt{2 \cos \alpha}, \sin x / 2), \\
u & =\sqrt{\mathrm{U}_{1}^{2} / 2 \mu \cos \alpha-u_{1}^{2}} \sinh \left(\xi_{0}-\chi\right) .
\end{aligned}
$$

This curve consists of a series of waves touching $\theta= \pm \alpha$ alternately and going to infinity along the line

given by

$$
\begin{array}{ll}
\chi=\xi_{0}, & p=\left(-\frac{d u}{d \theta}\right)_{u=0}=0, \\
\theta=\theta_{0}, & p=\left(-\frac{d u}{d \theta}\right)_{u=0}=0,
\end{array}
$$

$$
\sin \theta_{0} / 2=\sin \alpha / 2 \operatorname{sn}\left[\xi_{0} / \sqrt{2 \cos \alpha,} \sin \alpha / 2\right] .
$$

To obtain $\xi_{0}$ in terms of the velocities of projection $\mathrm{U}_{1} V_{1}$ and the coordinates of the point of the projection $r_{1} \theta_{1}$, we may remark that, if $\chi=\chi_{1}$, when $\theta=\theta_{1}$,

$$
\begin{aligned}
\sin \theta_{1} / 2 & =\sin \alpha / 2 \operatorname{su}\left(\psi_{1} / \sqrt{2} \cos \alpha, \sin \alpha / 2\right), \\
\tanh \overline{\xi_{0}-\chi_{1}} & =u_{1} \sqrt{2 \mu \cos \alpha / U_{1} .}
\end{aligned}
$$

Fig. 1.

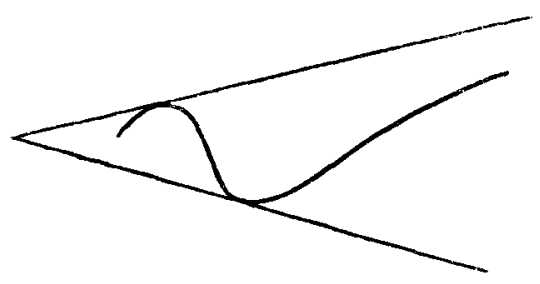

Thus

$$
\begin{aligned}
\xi_{0}=\sqrt{2 \cos \alpha} \operatorname{sn}^{-1} & \left(\sin \theta_{1} / 2 / \sin \alpha / 2\right) \\
& +\tanh ^{-1} u_{1} \sqrt{2 \mu \cos \alpha} / \mathrm{U}_{1} ;
\end{aligned}
$$

and hence the direction $\theta=\theta_{0}$ is given by

$$
\begin{aligned}
\sin \theta_{0} / 2= & \sin \alpha / 2 \operatorname{sn}\left[\operatorname{sn}^{-1}\left(\sin \theta_{1} / 2 / \sin \alpha / 2\right)\right. \\
& \left.+\frac{1}{\sqrt{2 \cos \alpha}} \tanh ^{-1} u_{1} \sqrt{2 \mu \cos \alpha} / U_{1}\right]
\end{aligned}
$$

A typical curve of this class is shown in fig. 1. 
In the case when $\mathrm{U}_{1}^{2} / 2 \mu m=v_{1}^{2}$, the equation stands in the form

and we get

$$
\frac{d u}{u \sqrt{\cos \alpha}}=-\frac{d \theta}{\sqrt{\cos \theta-\cos \alpha}}
$$

$$
\frac{1}{\sqrt{ } \cos \alpha} \log u / u_{1}=-\sqrt{ } 2\left(\psi-\psi_{1}\right)
$$

if $u=u_{1}$ when $\psi=\psi$, where

or

$$
\sin \theta / 2=\sin \alpha / 2 \operatorname{sn}(\psi, \sin \alpha / 2)
$$

$$
\begin{aligned}
\sin \theta / 2 & =\sin \alpha / 2 \operatorname{sn}\left[\frac{1}{\sqrt{2 \cos \alpha}} \log u_{1} / u+\psi_{1}\right] \\
& =\sin \alpha / 2 \operatorname{sn}\left[\psi_{1}+\frac{1}{\sqrt{2} \cos \alpha} \log r_{1} / r\right] .
\end{aligned}
$$

This curve oscillates between $\theta= \pm \alpha$, with $r$ continually increasing. As $r \rightarrow \infty \theta$ is indeterminate, and instead of tending to infinity along a certain line $\theta=\theta_{0}$ after a finite number of contacts with the lines $\alpha$ and $-c$, as in the previous case when $\mathrm{U}_{1}^{2} / 2 \mu \cos \alpha>v_{1}^{2}$, this curve oscillates between the rays $\pm \alpha$ and has no direction at infinity. A typical curve of this type is shown in fig. 2.

Fig. 2.

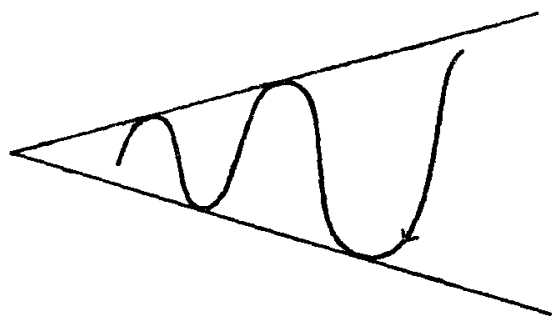

The case when $u_{1}^{2}-U_{1}^{2} / 2 \mu m=u_{2}^{2}>0$ remains. We bave

$$
\frac{d u}{\sqrt{\cos a \sqrt{u^{2}-u_{2}^{2}}}}=-\frac{d \theta}{\sqrt{\cos \theta-\cos \alpha}} .
$$

Putting $u=u_{2} \cosh \xi$, we get

$$
\frac{d u}{\sqrt{u^{2}-u_{2}^{2}}}=d \xi ;
$$

and consequently, if

$$
\sin \theta / 2=\sin \alpha / 2 \operatorname{sn}(\psi, \sin \alpha / 2)
$$


as before,

$$
\xi-\xi_{0}=-\sqrt{2 \cos c} \psi
$$

and the orbit in terms of a parameter $\psi$ and constants $\xi_{0}$ and $\alpha$ is

$$
\begin{aligned}
\sin \theta / 2 & =\sin (\alpha / 2) \operatorname{sn}(\psi, \sin \alpha / 2) \\
u & =\sqrt{ } u_{1}^{2}-U_{1}^{2} / 2 \mu \cos \alpha \cosh \left(\xi_{0}-\psi / \sqrt{ } 2 \cos \alpha\right) .
\end{aligned}
$$

It is evident that in this case, as $\psi$ increases from an initial value $\psi_{1}, u$ decreases until $\psi=\xi_{0} / \sqrt{2 \cos \alpha}$ : it then increases to infinity; while at the same time $\theta$ is going from the value $\theta_{1}$ through the cycle of values $(\alpha, 0,-\alpha, 0)$. A typical orbit of this kind is shown in fig. 3 .

Fig. 3.

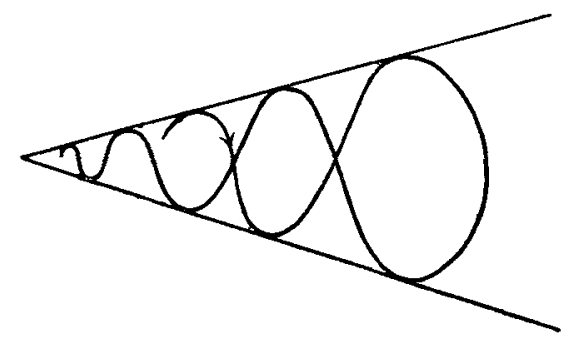

It is of interest to connect the constants in these equations with the constants given in the initial velocities at the point $r_{1} \theta_{1}$. We have, of course,

$$
\mathrm{V}_{1}^{2}=2 \mu u_{1}^{2}\left(\cos \theta_{1}-\cos \alpha\right)
$$

which gives $\alpha$ in terms of $v_{1} \theta_{1}$ and $\mathrm{V}_{1}$. Since $\theta=\theta_{1}$ when $r=r_{1}, \xi_{0}$ is given by the equations

$$
\sin \theta_{1} / 2=\sin \alpha / 2 \operatorname{sn}\left(\chi_{1} / \sqrt{2 \cos \alpha}, \sin \alpha / 2\right),
$$

$\tanh \overline{\xi_{0}-\chi_{1}}=V_{1} / \sqrt{ } 2 \mu u_{1}$;

so that

$$
\begin{aligned}
\xi_{0}=\sqrt{2 \cos \alpha} \operatorname{sn}^{-1} & \left(\sin \theta_{1} / 2 / \sin \alpha / 2\right) \\
& +\tanh ^{-1} \mathrm{U}_{\mathrm{l}} / \sqrt{2 \mu u_{1}} \sqrt{\cos \alpha .}
\end{aligned}
$$

It is perhaps hardly necessary to get the equation in the case of projection towards the origin, since the analysis is entirely similar to that required in the cases when projection is along the axis, and the geometrical characteristics of the curves obtained have already been indicated in the diagram. For example, the part of the curve (fig. 3) in which the particle is approaching the origin is a typical curve of the case when projection is towards the doublet. 
Motions which are restricted to half the Plane, including one Periodic Orbit.

We have now considered the cases when

$$
0 \leq 2 \mu \cos \theta_{1}-h^{2} \leq 1 .
$$

We have considered in detail the boundary case

$$
2 \mu \cos \theta_{1}-h_{1}^{2}=0,
$$

in which motion is restricted to the line $\theta=0$.

The particular features of the case

$$
2 \mu \cos \theta_{l}-l^{2}=0, \quad l^{2}=2 \mu \cos \theta,
$$

also call for attention. Here the motion is restricted to the positive side of the plane, given by

$$
-\frac{\pi}{2} \leq \theta \leq \frac{\pi}{2}
$$

Referring to the equation

we get in this case

$$
\ddot{r}-r \theta^{2}=\mathrm{R},
$$

$$
\ddot{r}=0, \quad \dot{r}=\mathrm{U}_{1} ;
$$

so that the radial velocity is constant. Given then an outward radial velocity initially, the curve touches the lines $\pm \pi / 2$ alternately, and the distance at any time from the origin of a particle describing it is given by

$$
r-r_{1}=\mathrm{U}_{1}\left(t-t_{1}\right)
$$

if it is projected from $r_{1}$ with radial velocity $\mathrm{U}_{1}$ at $t_{1}$. If $\dot{r}=-\mathrm{U}_{1}$, the particle approaches the doublet with constant radial velocity along a wave path which alternately grazes $\theta= \pm \pi / 2$.

The equation to the orbit if $i=U_{1}>0$ results from the equation

or

$$
-d u / u d \theta=d r / r d \theta=\mathrm{U}_{1} / r \dot{\theta}=\frac{\mathrm{U}_{1}}{u \sqrt{2 \mu \sqrt{\cos } \theta}}
$$

in the form

$$
d u / d \theta=-\frac{U_{1}}{\sqrt{2 \mu}}: \sqrt{\cos \theta}
$$

$$
\begin{aligned}
& \sin \theta / 2=\frac{1}{\sqrt{2}} \sin (\psi, 1 / \sqrt{ } 2), \\
& u-u_{1}=-\frac{U_{1}}{\sqrt{2 \mu}} \cdot \sqrt{ } 2\left(\psi-\psi_{1}\right)
\end{aligned}
$$


if $u=u_{1}$ when $\psi=\psi_{1}$. Thus

and

$$
\sin \theta / 2=1 / \sqrt{ } 2 \operatorname{sn}\left[\psi_{1}-\frac{\mathrm{U}_{1}}{\sqrt{\mu}}\left(u-u_{1}\right)\right]
$$

$$
\sin \theta_{1} / 2=1 / \sqrt{ } 2 \sin \psi_{1} \text {. }
$$

The equation connecting $\theta$ and $t$ can easily be obtained in the form

$\sin \theta / 2=1 / \sqrt{ } 2 \operatorname{sn}\left[\operatorname{sn}^{-1}\left(\sqrt{ } 2 \sin \theta_{1} / 2\right)+\frac{U_{1}^{2}}{\sqrt{ } \mu} \cdot \frac{\left(t-t_{1}\right)}{r_{1}\left(r_{1}+U_{1} \overline{t-t_{1}}\right)}\right]$.

In the case of $\dot{r}$ being negative originally and equal to $-\mathrm{U}_{1}$, we have

$$
r-r_{1}=\mathrm{U}_{1}\left(t_{1}-t\right) \text {, }
$$

and progress is towards the origin along a curve waving between $\theta= \pm \pi / 2$ and cutting the line $\theta=0$ between each two consecutive grazings of these boundary lines. It may possibly be of interest to remark that the reciprocals of the distances of the various points on the rays $\theta=\alpha, \theta=0$, or $\theta=-\alpha$ are in arithmetic progression with common difference $\sqrt{ } 2 \mu \mathrm{K} 1 / \sqrt{ } 2 / u_{1}$.

A case of special interest occurs, however, when the radial velocity originally is zero. The curve will then be merely the part of the circle $r=r_{1}$ for which

$$
-\pi / 2 \leq \theta \leq \pi / 2 \text {. }
$$

The transverse velocity is given by

$$
V^{2}=2 \mu \cos \theta / r^{2} \text {. }
$$

Thus, if a particle is given a transverse velocity

$$
\mathrm{V}_{1}=\sqrt{2 \mu \cos \theta_{1}} / r_{1}
$$

at the point $\theta_{1} r_{1}$ and no radial velocity, it will describe the semicircle $r=r_{1},|\theta| \leq \pi / 2$ perpetually.

The periodic time is easily given by

$$
\begin{aligned}
\frac{1}{4} \mathrm{~T}=\int_{0}^{\pi / 2} r_{1} \frac{d \theta}{\mathrm{V}} & =\frac{r_{1}^{2}}{\sqrt{2 \mu}} \int_{0}^{\pi / 2} \frac{d \theta}{\sqrt{\cos \theta}} \\
& =\frac{r_{1}^{2}}{\sqrt{ } 2 \mu} \int_{0}^{\pi / 2} \frac{d \theta}{\sqrt{1-2 \sin ^{2} \theta / 2}}=\frac{r_{1}^{2}}{\sqrt{2 \mu}} \mathrm{K}(\sqrt{ } 2) \\
& =-\frac{r_{1}^{2}}{\sqrt{2 \mu}} \cdot \sqrt{ } 2 \mathrm{~K}(1 \sqrt{ } / 2) .
\end{aligned}
$$


We have now, therefore, established the existence of a periodic orbit in the field of force

$$
\mathrm{V}=-\mu u^{2} \cos \theta \text {, }
$$

viz. the semicircle $r=r_{1}$, in which the periodic time is $4 r_{1}{ }^{2} \mathrm{~K}(1 / \sqrt{ } 2) / \sqrt{ } \mu$. The path of a particle is therefore this periodic orbit when the conditions of projection at $r_{1} \theta_{1}$ give

$$
\mathrm{U}_{1}=0, \quad r_{1}^{2} V_{1}^{2}=2 \mu \cos \theta_{1},
$$

and only under these conditions.

It is further possible to deduce from our general analysis that when the transverse velocity of projection satisfies the conditions

$$
0 \leq 2 \mu \cos \theta_{1}-r_{1}^{2} V_{1}{ }^{2} \leq 2 \mu
$$

this semicircular path is the only periodic orbit possible.

We next consider the case when

Putting

$$
0<h_{1}^{2}-2 \mu \cos \theta_{1} \leq 2 \mu \text {. }
$$

$$
h_{1}^{2}-2 \mu \cos \theta_{1}=-2 \mu \cos \alpha=2 \mu m>0,
$$

we see at once that motion must be restricted to the sector

$$
-\alpha \leq \theta \leq \alpha,
$$

in which $\alpha>\pi / 2$. In this case the $(r, t)$ equation is

giving

$$
\ddot{v}=2 \mu \mathrm{m} / \mathrm{r}^{3} \text {, }
$$

$$
i^{2}=\mathrm{U}_{1}{ }^{2}+2 \mu m / r_{1}{ }^{2}-2 \mu m / r^{2} .
$$

If $i$ is originally positive or zero, $\dot{r}$ increases steadily to the value $\checkmark\left(U_{1}^{2}+2 \mu m / r_{1}^{2}\right)$ at infinity; while if it is originally negative, it decreases in absolute magnitude until $r=r_{2}$, where

$$
1 / r_{2}^{2}=1 / r_{2}{ }^{2}+U_{1}^{2} / 2 \mu m
$$

and then changes sign and increases steadily. We may integrate the equation in the form

$$
t-t_{1}=r_{2}\left(\sqrt{r_{1}^{2}-r_{2}^{2}}-\sqrt{r^{2}-r_{2}^{2}}\right) / \sqrt{2 \mu m} \text { when } U_{1}<0,
$$

and in the form

$$
t-t_{1}=r_{2} \sqrt{r^{2}-r_{2}^{2}} / \sqrt{2 \mu m} \text { if } \mathrm{U}_{1}>0 \text {. }
$$

Whatever, then, the initial radial velocity the particle proceeds either directly or after an interval

$$
2 r_{2} \sqrt{r_{1}^{2}-r_{2}^{2}} / \sqrt{2 \mu m}
$$

to increase its distance from the origin at an increasing rate. 
Orbits in the Field of a Doublet.

At the same time, the variation of $\theta$ can be obtained from the equations

$$
\begin{aligned}
\sin \theta / 2 & =\sqrt{\frac{m+1}{2}} \operatorname{sn}\left(\phi, \sqrt{\left.\frac{m+1}{2}\right)}\right. \\
r & =r_{1} \sec \left(\sqrt{2 m}\left(\phi-\phi_{1}\right)\right),
\end{aligned}
$$

or if the relation between $\theta$ and $t$ is more convenient, from the equation

$$
t-t_{1}=\left(r_{1}^{2} / \sqrt{2 \mu m}\right) \tan \left(\sqrt{2 m}\left(\phi-\phi_{1}\right)\right) .
$$

These equations can be deduced from the relation

$$
\mathrm{U} / \mathrm{V}=\boldsymbol{r} d \theta / d r
$$

as before. The path therefore, in general, waves backwards and forwards between $\theta= \pm \alpha$ a finite number of times (which may be zero), and has the asymptote

given by

$$
\theta=\theta_{0}, \quad p=(-d u / d \theta)_{u=0}
$$

$$
\sin \theta_{0} / 2=\sqrt{\frac{m+1}{2}} \operatorname{sn}\left(\phi_{1}+\pi / 2 \sqrt{2 m}\right) .
$$

A typical orbit is appended in fig. 4.

Fig. 4.

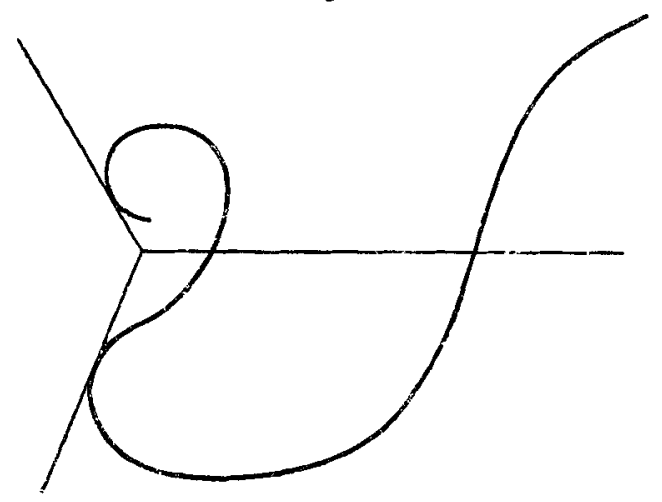

There is, however, one particular case. If

$$
h_{1}^{2}-2 \mu \cos \theta_{1}=2 \mu,
$$

then $\alpha=\pi$ and the transverse velocity disappears when the line $\theta=\pi$ is reached. Unless therefore the particle goes off Phil. May. Ser. 6. Vol. 43. No. 257. May 1922. 3 T 
to infinity before reaching the line $\theta=\pi$, it will approach the line tangentially and pursue the path solely along $\mathrm{t} !$ is line.

\section{Characteristics of Orbits not restricted to a Sector of the Plane.}

The final case to be considered is when

$$
h_{1}^{2}-2 \mu \cos \theta_{1}=2 \mu m>2 \mu .
$$

In this case, since

and

$$
h^{2}=2 \mu(m+\cos \theta)
$$

$$
\mathrm{V}^{2}=2 \mu(m+\cos \theta) / r^{2},
$$

the transverse velocity never vanishes in the finite part of the plane. The equation to the orbit in terms of $a$ parameter $\phi$ is still given by

$$
\sin \theta / 2=\sqrt{\frac{m+1}{2}} \operatorname{sn}\left(\phi, \sqrt{\frac{m+1}{2}}\right),
$$

which is now more conveniently put in the form

and

$$
\sin \theta / 2=\operatorname{sn}\left(\sqrt{\frac{m+1}{2}} \phi, \sqrt{\frac{2}{m+1}}\right)
$$

$$
\begin{aligned}
r & =r_{1} \sec \sqrt{2 m}\left(\phi-\phi_{1}\right), \\
\mathrm{U} & =\sqrt{ } 2 \mu m \sqrt{1 / r_{1}^{2}-1 / r^{2}+\mathrm{U}_{1}^{2} / 2 \mu m} .
\end{aligned}
$$

The particle goes to infinity, as before, arriving there with the velocity $\sqrt{ } 2 \mu m \sqrt{1 / r^{2}+\mathrm{U}_{1}^{2} / 2 \mu m}$, and with $\sqrt{ } 2 m\left(\phi-\phi_{1}\right)$ $=\pi / 2$. Now the equation giving $\theta$ in terms of $\phi$ will show what angular distance from $\theta_{1}$ is described before the purticle reaches infinity. It is evident that as $m$ increases this angular distarce increases, giving in the limiting case an infinite angular distance. The orbit therefore, in this general case, is a curve described with $r$ and $\theta$ increasing together and circumscribing the origin an increasing number of times as $m$ increases, and tending to intinity along the line $\theta=\theta_{0}, p=\left(-\frac{d u}{d \theta}\right)_{u=0}$. In the limiting case when $m$ is infinite, the path of the curve circumscribes the origin for ever, and only has the circle at infinity as asymptote.

If the particle is projected with negative or zero radial 
velocity, and the transverse velocity still satisfies the conditions

$$
r_{1}^{2} \nabla_{1}^{2}-2 \mu \cos \theta_{1}>2 \mu,
$$

the particle approaches the origin to a distance $r=r_{2}$ given by

$$
1 / r_{2}^{2}=1 / r_{1}^{2}+U_{1}^{2} / 2 \mu m
$$

in times given by

$$
t-t_{1}=r_{2}\left(\sqrt{r_{1}^{2}-r_{2}^{2}}-\sqrt{r^{2}-r_{2}^{2}}\right) / \sqrt{2 \mu m},
$$

and then recedes from the origin according to the law

$$
t-t_{1}=r_{2} \sqrt{r^{2}-r_{2}^{2}} / \sqrt{2 \mu m .}
$$

While the particle is first approaching and then receding from the origin, the variation of $\theta$ with $t$ is given by

$$
\begin{aligned}
\sin \theta / 2 & =\operatorname{sn}\left(\sqrt{\frac{m+1}{2}} \phi, \sqrt{m+1}\right), \\
t-t_{1} & =r_{1}^{2} / \sqrt{2 \mu m} \tan \sqrt{2 m}\left(\phi-\phi_{1}\right) ;
\end{aligned}
$$

so that the orbit circumscribes the origin until that value of $\theta$ is reached for which $\left(\phi-\phi_{1}\right) \sqrt{z m}=\pi / 2$.

A typical orbit is shown in fig. 5 .

Fig. 5.

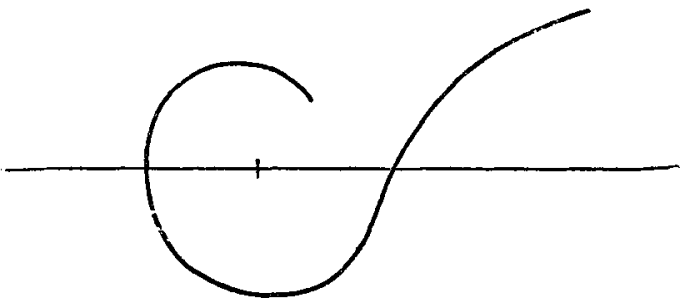

As in the previous cases, if the initial radial velocity is towards the origin there is a finite excursion towards the origin before the ralial velocity becomes positive and increases steadily as time goes on.

\section{Summary.}

The general characteristics of the orbits in the field of a doublet may be summed up as follows :-

(a) The only periodic orbits are the semicircles

$$
r=r_{1}, \quad-\pi / 2 \leq \theta \leq \pi / 2,
$$

fur various values of $r_{1}$. The corresponding periodic time is

$$
r_{1}^{2} \mathrm{~K}(1 / \sqrt{ } 2) / \sqrt{ } \mu \text {. }
$$


These orbits are described when and only when the conditions of projection at the point $\left(r_{1} \theta_{1}\right)$ are such that the radial velocity is zero and the transverse velocity. $V_{1}$ is given by

$$
r_{1}^{2} V_{1}^{2}=2 \mu \cos \theta_{1}
$$

Included under these conditions is the case when a particle is placed at rest on either of the lines $\theta=\pi / 2, \theta=-\pi / 2$, at any non-zero non-infinite distance $r_{1}$ from the doublet. The orbit will then be the appropriate semicircle, and the periodic time will be as above.

(b) When the transverse velocity of projection is such that

$$
0 \leq 2 \mu \cos \theta_{1}-r_{1}{ }^{2} V_{1}{ }^{2}=2 \mu m \leq 2 \mu,
$$

the particle only leaves the system if $U_{1}$, the radial velocity, is greater than ur equal to $\sqrt{2 \mu m} / r_{1}$. The orbit touches the rays $\theta= \pm \alpha$ alternately, where $\cos \alpha=m$, and proceeds to infinity with a certain line

$$
\theta=\theta_{0}, \quad p=(-d v / d \theta)_{u=0},
$$

as asymptote, if $\mathrm{U}_{1}>\sqrt{2 \mu m} / r_{1}$. If $\mathrm{U}_{1}=\sqrt{2 \mu m} / r_{1}$, the orbit touches the rays alternately for ever and does not have a linear asymptote.

If, however, $\mathrm{U}_{1}$ does not attain this critical velocity, the orbit touches the rays alternately and keeping to the sector $|\theta| \leq \alpha$, first recedes from the doublet if $\mathrm{U}_{1}>0$, and then approaches the doublet converging on it, through a series of waves of decreasing amplitude, which touch the lines which bound it. If $\mathrm{U}_{1}$ is negative, the orbic has similar characteristics, the excursion away from the origin being now deleted. However, whatever the value of $\mathrm{U}_{1}$, under the condition

$$
0 \leq 2 \mu \cos \theta_{1}-r_{1}^{2} V_{1}^{2} \leq 2 \mu
$$

the orbit is confined between the two rays $\theta= \pm \alpha$, where $\alpha \leq \pi / 2$, and

$$
2 \mu \cos \theta_{1}-r_{1}{ }^{2} V_{1}{ }^{2}=2 \mu \cos \alpha,
$$

and is of wave form converging on or diverging from the doublet, the amplitude of the wave varying in such a way that the curve touches the lines which bound it.

(c) When the transverse velocity of projection is such that

$$
0<r_{1}^{2} \mathrm{~V}_{1}^{2}-2 \mu \cos \theta_{1} \leq z \mu,
$$

the orbit, whatever $U_{1}$ may be, whether it is negative or positive, goes to infinity and the particle describing it recedes from the origin-alter a finite excursion towards the doublet if $U_{1}$ is negative-at a steadily increasing rate, 
and it takes an infinite time to leave the system. The path again lies between bounding rays $\theta= \pm \alpha$, but

$$
\cos \odot=\cos \theta_{1}-r_{1}^{2} V_{1}^{2} / 2 \mu,
$$

and therefore $\alpha$ is greater than $\pi / 2$; the orbit is therefore confined to an oblique-angled sector of the plane. The path in general touches the bounding rays a finite number of times or not at all and has a certain straight line as asymptote. No velocity of projection towards the origin, however large, enables the particle to retch the doublet, or prevents it continually increasing its distance from the origin, possibly after it has approached within a certain distance of the doublet.

(d) When the transverse velocity is such that

$$
2 \mu<r_{1}^{2} / V_{1}^{2}-2 \mu \cos \theta_{1}
$$

the orbit is not restricted to any sector of the plane. The particle ayain approaches the doublet if the radial velocity of projection is towards the origin, but it never reaches it: in this case it ultimately recedes from the doublet. When the radial velocity of projection is positive it straightway recedes from the doublet. While the distance of the particle of the doublet is changing in this way, the angular distance of the particle from its initial position steadily increases, and it finally proceeds to infinity in the direction of a certain line, after a finite number of circumscribings of the origin. As

$$
r_{1}^{2} V_{1}-2 \mu \cos \theta_{1}
$$

increases, the orbit becomes more and more like the orbit in the limiting case when

$$
r_{1}^{2} V_{1}^{2}-2 \mu \cos \theta_{1}
$$

is infinite; in this case the particle circumscribes the doublet in the same sense, for ever, while its distance from the doublet increases without limit.

The main interest of the results lies probably in the electrification of neutral atoms in a vacuum tube-the neutral atom being taken, as usual, in its first approximation as a doublet. All the formulæ deduced involve, essentially, a critical velocity of an electron, which determines whether it leaves the atom in whose proximity it finds itself, or whether it stays. On the Quantum Theory, the criteria which determine the actual capture of such an electron by an atom have not yet been defined, and the fact that an electron "stays," according to this analysis, 


\section{Dr. E. H. Kennard on a Simplified Proof for}

does not perbaps of itself involve its capture and retention as a constituent part of a negatively charged atom. Nevertheless there must be a correspondence and perhaps a rough proportionality between the two classes of pbenomena-fres electrons with a velocity distribution about the critical value, and the number of negatively charged atoms found in tubes under the conditions of discharge. It would seem that an experimental estimate of the number of such atoms. is desirable in relation to the velocity distribution in the free electrons of which we have some definite knowledge. The Quantum Theory of atoms itself, proceeding as it does by the elaboration of successive hypotheses, still requires a hypothesis regarding the capture of electrons and the formation of negatively charged atoms. It would be of interest if it could be shown that a stray electron which by the foregoing analysis could not leave the system really became "bound" as a constituent part of the atomic : we may, indeed, perhaps anticipate that this phonomenon usually occurs.

CIV. On a Simplified Proof for the Retarded Potentials and Huyghens's Priuciple. By E. H. KenNard, Ph.D.*

THE proof usually given in deducing the retarded potentials and Huyghens's Principle seems to the author, as it must to many physicists, peculiarly abstract and indirect. This objection is only partly met in Professor Mason's $\dagger$ modification. The following proof seems to be at least as short and as rigorous as any other, while, at the same time, it seems more natural and easier for a physicist to follow.

\section{§. New. Proof.}

The scalar potential $\phi$ satisfies the differential equation

$$
\frac{1}{c^{2}} \frac{\partial^{2} \phi}{\partial t^{2}}=\nabla^{2} \phi+4 \pi \rho, \ldots . . . . .
$$

where $\rho=$ density of electricity (the units being "ordinary").

To find $\phi$ at time $t$ at any point $\mathrm{P}$, let us surround $\mathrm{P}$ by any closed surface $S$ and then, following Abraham, let us

* Communicated by the Author.

† Max Mason, Phys. Rev. vol. xv. p. 312 (1920). 